\title{
GUERRA Y CANIBALISMO EN EL VALLE DEL RIO CAUCA EN LA EPOCA DE LA CONQUISTA ESPAÑOLA
}

Luis Gonzalo Jaramillo E.*

* Instituto Colombiano de Antropología.

Revista Colombiana de Antropología, vol XXXII. 1995 
At the time of the conquest the Spaniards reported a high incidence of warfare and cannibalism among the groups of the Cauca Valley. This article points out that one of the main problems with recent interpretations of warfare and cannibalism is the assumption that the incidence of these practices as reported by the Spaniards reflects the actual state of indigenous affairs. This attitude takes no account of the impact of the Spanish presence in terms of the creation of new political, military and social conditions. While both aspects (warfare and cannibalism) are discussed, the article concentrates on warfare insofar as it relates to social organisation. The development of archaeological investigation of settlement patterns is proposed as a research strategy to discover independent strands of evidence to promote better understanding of the sociopolitical and economic conditions under which the system of warfare and cannibalism operated in prehispanic times. The Anserma, Quimbaya and Arma groups are discussed from this perspective.

\section{INTRODUCCION ${ }^{1}$}

En la literatura antropológica, la región del valle del río Cauca es mejor conocida por la presencia de un estado de guerra y canibalismo crónicos entre los grupos que la habitaban en la época de la conquista española (Restrepo 1912; Eckert 1945; Hernández 1948; Trimborn 1949; Duque Gómez 1963; Friede 1982; Schorr 1968; Arocha 1987; Pineda 1987; Carneiro 1990). Aunque la guerra y el canibalismo son dos fenómenos independientes ${ }^{2}$, en el caso

El autor quiere expresar un sincero reconocimiento de gratitud por los comentarios a una versión anterior de este documento a Robert D. Drennan, Jeff Blick, Robert Kruger, Carl Langebaek, Santiago Mora y Leonor Herrera. Así mismo a Norberto Ballesteros por sus sugerencias de estilo.

2 Puesto que estos tópicos han sido objeto de discusiones extensas y detalladas en el ámbito de la literatura antropológica, no se intentará aqui una discusión exhaustiva. Los siguientes trabajos se reseñan como referencia.básica en el tema: Brown y Tuzin, eds. 1983; Harrison 1948; Arens 1979; Tannahill 1975; Fried et al. eds. 1968; Harner 1972; Ferguson 1984; Chagnon 1983; Harris 1977; Blick 1987; Schorr 1970. 
del valle del río Cauca éstos aparecen profundamente interrelacionados. De hecho, las referencias más dramáticas sobre la intensidad de la práctica canibal se enmarcan en la descripción de episodios de guerra.

Estos dos fenómenos han sido analizados desde perspectivas diferentes. Arocha $(1987: 32,34,35)$, por ejemplo, argumenta que la guerra resultaba de la competencia entre grupos por medioambientes preferidos y restringidos, y que el canibalismo operaba como mecanismo para enfrentar el problema de suministro de proteínas, derivado de una situación de presión demográfica sobre los" recursos. Schorr (1968:458) ve la guerra y el canibalismo, como partes de un mecanismo de nivelación para aliviar riesgos de subsistencia "... causados por deficiencias en la producción como resultado de sequías, plagas, inundaciones y otras variaciones estacionales" (véase también Schorr 1965:123 y 1970).

Carneiro (1990:193,194), quien considera los niveles crónicos de guerra reportados en las crónicas como una expresión típica de la trayectoria de desarrollo de los cacicazgos, concluye que la principal razón para la guerra fue la captura de prisioneros, la mayor parte de los cuales eran comídos en el contexto de ceremonias mágico-religiosas.

Otro punto de vista es el de Pineda (1987) quien acepta la existencia de la práctica canibal como parte de un complejo mágico-religioso, pero propone un análisis crítico de las condiciones generales en las cuales las crónicas fueron escritas para explorar, de esta manera, las circunstancias que explicarian los niveles de eánibalismo reportados. En este sentido, Pineda considera que esas condiciones fueron las de un encuentro violento entre culturas (indigena versus española) que crea una "cultura del terror" y un "espacio de muerte" (Pineda 1987:93 citando a Tausing 1987) del cual ambos grupos participaban igualmente.

- El propósito de este artículo es discutir algunos de los problemas que presentan las actuales interpretaciones, concentrándose principalmente en el aspecto de la guerra. Específicamente, se discutirá en primer lugar, el argumento que considera que los niveles de guerra reportados por los españoles son una reflexión objetiva de los patrones de guerra en tiempos prehispánicos, puesto que éstos describen la guerra cuando transcurría aún "...bajo condiciones esencialmente aborígenes" (Carneiro 1990:192). En segundo lugar, se discutirá el argumento que .ve el canibalismo como una manera de aliviar deficiencias en el suministro de proteínas (Arocha 1987; Bruhns 1981:8). 
El argumento propuesto aquí es que uno de los mayores problemas que enfrenta cualquier intento por proveer explicaciones causales para estas prácticas es - además de la necesidad de una lectura crítica de las crónicas - la falta de parámetros claros para medir los niveles de población indígena, la organización sociopolítica y disponibilidad de recursos, así

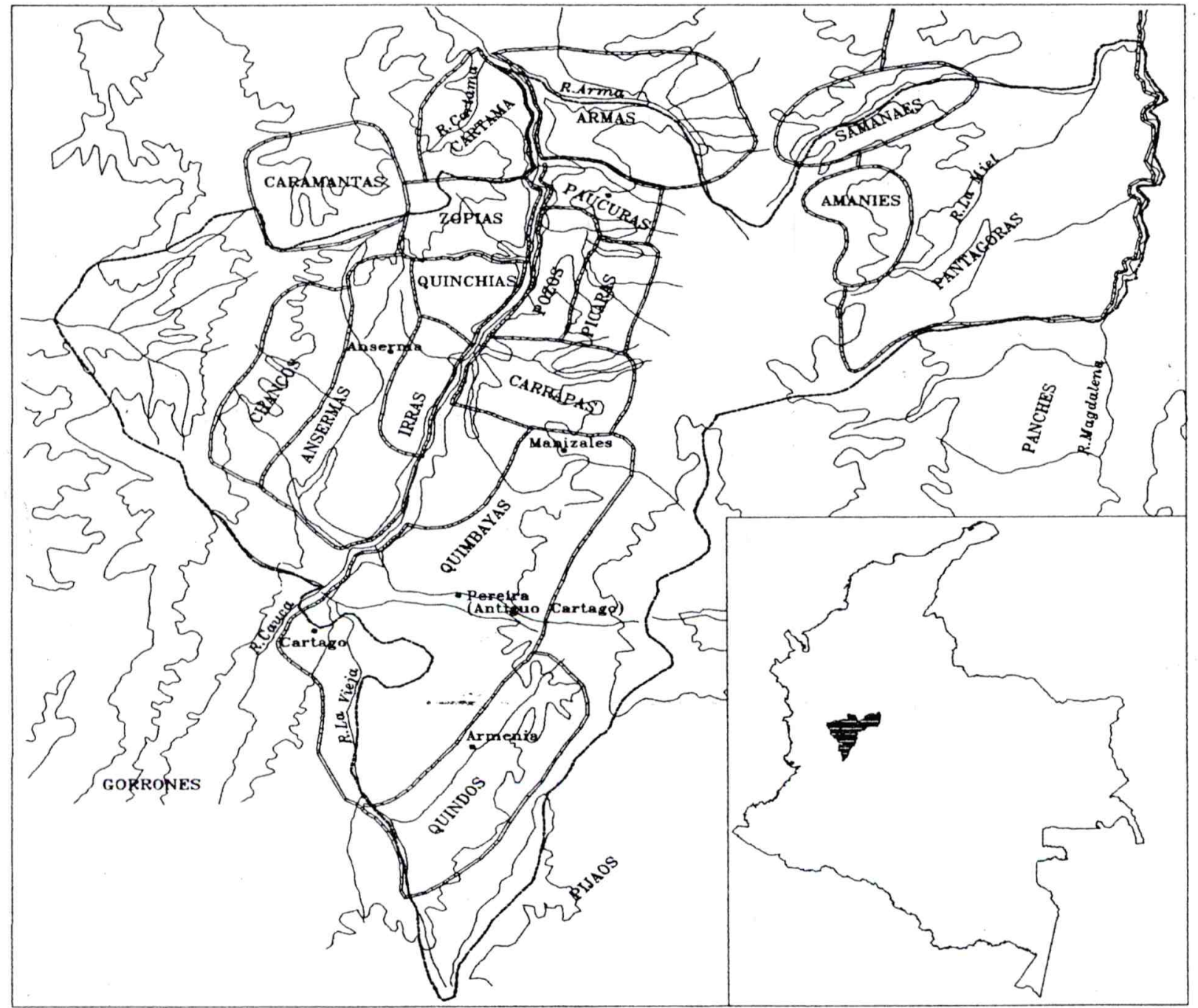

El valle medio del rio Cauca y la ubicación de los grupos mencionados en el texto (tomado del el mapa "Grupos indigenas que poblaban el antiguo departamento de caldas en el siglo XVI", Duque Gómez, 1970). 
como la capacidad de producción de estas sociedades. Se argumentará que una fuente importante de problemas emana de la casi absoluta dependencia en las crónicas para evaluar estos aspectos, dado que existen pocas crónicas de testigos oculares así como por las inconsistencias que éstas presentan.

El desarrollo de investigaciones arqueológicas de patrones de asentamiento se propone como estrategia para producir líneas de evidencia independiente, que permitan evaluar la información proporcionada por las crónicas. Esta estrategia puede proveer, adicionalmente, parámetros para evaluar otros aspectos tales como la densidad de población y por extensión, argumentos sobre presión demográfica.

Dada la extensión del valle del río Cauca, la discusión se limitará a la región y a los grupos de la zona media del valle. En primer lugar se hará una breve reseña histórica de la zona como marco de referencia. Luego se discutirá el problema que resulta de una aceptación literal de las crónicas relacionadas con la guerra y el canibalismo, así como las explicaciones ofrecidas en relación con estos aspectos. Finalmente se analiza el caso de los Ansermas, Quimbayas y Armas para ilustrar cómo los estudios arqueológicos de patrones de asentamiento pueden contribuir al esclarecimiento de estos aspectos.

\section{EL VALLE DEL RIO CAUCA EN PERSPECTIVA}

La cuenca hidrográfica del río Cauca, delimitada por la cordillera Central y la cordillera Occidental, presenta elevaciones que varian entre 205 metros sobre el nivel del mar en las cercanías de Mompox y 5590 en el volcán Nevado del Ruiz. Esta variación altitudinal determina la presencia de diferentes zonas ecológicas, ya que en la latitud ecuatorial, el clima es determinado por la altitud.

Las primeras expediciones españolas en esta zona, alrededor de 153040 (véase Trimborn 1949:36-42 y Duque Gómez 1963, 1967, 1970 para una reseña detallada), indican que la región tenía una población relativamente densa; Trimborn (1949:63) considera que unos 44 grupos habitaban la región. Jaramillo (1964:284) ha estimado la población total entre las cordilleras Central y Occidental en unos 250,000 individuos, mientras que Llanos (1981:38) estima ésta en unas 500,000 personas. Tovar (1992:56) estima una población de más de tres millones de habitantes en la zona andina del Occidente colombiano. 
Los estimativos parciales dentro de la región, aunque también variables, son útiles para obtener una visión de magnitudes relativas. Friede (1963:308) estima la población de los Quimbayas alrededor del año 1540 en unas 60.000 personas, con una densidad de 20 individuos por kilómetro cuadrado. Jaramillo (1964:256), por su parte, estima ésta en unos 20.000 individuos (ver también Friede 1982:23-24; Tovar 1970:65-91; 1992:64; Padilla 1977:43); Llanos $(1981: 32,36)$ estima la población del área de Popayán en unos 180.000 habitantes, con una densidad de 24 por kilómetro cuadrado.

Aunque es necesaria una estimación más precisa de los niveles de población en la época de la conquista, así como también lo es la del proceso de decrecimiento del contacto, parece claro que este último fue muy rápido y dramático. Por ejemplo, los Quimbayas fueron casi exterminados al cabo de unos 80 años del contacto (Friede 1963:307). Para el área de Popayán existen figuras similares de disminución: de una población estimada en 180.000 personas en el año de 1537, sólo quedaban 7860 alrededor de 1606 (Llanos 1981:30; ver también Gerónimo de Escobar [1582] 1884).

Poco después de su arribo, los españoles entendieron que el decrecimiento de la población indigena amenazaba sus aspiraciones económicas. Por esta razón, comenzaron a intervenir en las comunidades indígenas y alterar sus sistemas sociopolíticos no sólo mediante el desplazamiento de hombres y familias, sino también con el traslado de comunidades completas de un lugar a otro (Friede 1963:310) para así ajustar y compensar la disminución de la mano de obra que resultaba tanto de los combates entre indígenas y españoles, como de la acción de epidemias y sobre-explotación. El florecimiento, decaimiento y/o reubicación de las villas españolas durante este período, refleja los efectos y cambios que se operaban en las esferas socio-política y económica de la región en general (ver Tovar 1992:55, 57 y 68).

El rápido decrecimiento de la población indígena como resultado de esta combinación de factores, explica hasta cierto punto, la escasez de documentos relacionados con estos grupos ${ }^{3}$. No obstante, lo que se debe enfatizar aquí es, que debido a este proceso de rápida disminución de la población, los documentos más confiables para una reconstrucción de los patrones culturales y sociopolíticos son aquellos anteriores al año de 1.560; el número de éstos, no

3 Debe señalarse que muchos documentos se han perdido debido a innumerables razones, tales como incendios, descuido, etc, y que archivos tales como los de Sevilla (España), el Archivo Nacional (Santa Fe de Bogotá), el Archivo Central del Cauca (Popayán) bien podrian contener materiales relevantes todavía no conocidos. 
obstante, es muy pequeño (ver Robledo [sf.] 1865, Cieza [1550] 1985a, 1985b , $^{4}$ Sardella [sf.] 1864, Sarmiento [1540] 1864). En este sentido se puede anotar que mientras una relación anónima fechada en los años 1559-60 claramente establece que en la región de Caramanta-Anserma no existen caciques ó señores, las primeras descripciones hechas por Robledo alrededor de 1539 establecen la presencia de éstos y proporcionan información adicional sobre su estatus particular, así como sobre los alcances de su poder (Pineda 1987:87).

De manera general, las circunstancias ya mencionadas sobre el proceso de conquista, sugieren de antemano algunos de los problemas que se presentan al intentar reconstruir los niveles de organización sociopolítica de estos grupos tomando como base exclusiva las crónicas españolas. Este aspecto será discutido con más detalles en la parte final.

\section{LA GUERRA Y LOS DOCUMENTOS ESPAÑOLES: EVALUACION DE LA EVIDENCIA}

La presencia de un estado de guerra crónico entre los grupos del valle del río Cauca se ha convertido en uno de sus elementos más característicos, debido a su prevalencia tanto en las crónicas como en los subsecuentes estudios etnohistóricos y antropológicos. El concepto de un estado de guerra crónico se origina en las crónicas mismas, y es tan persistente en ellas, que sólo las referencias hechas a la presencia de oro podrían crear un equilibrio entre El Dora$d o^{5}$ que los españoles buscaban y el territorio infernal - lleno de caníbalesque describieron. Los investigadores modernos también han contribuido a fomentar y mantener la noción o idea de un estado de guerra "universal, agudo y sin fin" (Carneiro 1990:193) debido a la aceptación de las crónicas en una forma casi literal, así como por la tendencia a hacer generalizaciones basadas en tales reportes ${ }^{6}$.

4 Dada las frecuentes referencias a este autor, en adelante sólo se incluirá el año de la publicación que se utiliza , así: 1985a para "La Crónica del Perú" y 1985b para "Las Guerras Civiles Peruanas". Cada referencia está seguida del Capitulo y la página.

5 El nombre de Pais de Arvi (Cieza [1550] 1985:T-I, C-XXII:34; T-II, C-VII: 166); Sardella 1864:303, 307) se utilizó para designar una región localizada en la parte este de la cordillera central, que podría corresponder al territorio de los Muiscas (Friede 1963:43) y en donde se origina la leyenda de EL Dorado (véase Bray 1979).

6 Por ejemplo, Carneiro (1990:194), para demostrar que los indígenas del valle del Cauca se encontraban “...en pie de guerra permanentemente...", anota que Trimborn (1949) escribió sobre estos grupos que, " Cuando ellos están plantando o trabajando los cam- 
Las tendencias anteriormente descritas, figuran entre los problemas más críticos que se observan en las explicaciones recientes sobre la naturaleza de las sociedades del valle del Cauca, ya que las crónicas crean una imagen sesgada de condiciones homogéneas, la cual restringe nuestro entendimiento de estos grupos, de sus relaciones y, por ende, de la escala o magnitud de la guerra en tiempos prehispánicos. Aunque la elaboración de explicaciones de carácter general sobre procesos culturales es una meta, está debe ser la sintesis de estudios detallados y sistemáticos de casos particulares. Desafortunadamente, tal y como se mostrará a continuación, en muchos aspectos, la información disponible es todavía deficiente ${ }^{7}$.

En general, tenemos que la razón fundamental por la cual los reportes españoles son aceptados de una forma casi literal, es la creencia que los españoles, en verdad, "observaron y describieron [la guerra] en considerable detalle mientras ésta se llevaba a cabo bajo condiciones esencialmente aborígenes," tal y como Carneiro (1990:192) lo ha observado recientemente. No obstante, este planteamiento, el cual es la piedra angular de su análisis, es problemático. De hecho, las crónicas son una fuente dudosa para describir las condiciones de guerra en tiempos prehispánicos, ya que ellas no provienen de reportes hechos por observadores neutrales que describen

pos, en una mano sostienen el palo de rozar y en la otra una lanza para pelear". Este comentario, no obstante, fue hecho por Cieza ([1550] 1985:T-I,C-XXI:32) y se refiere específicamente a los Pozos, tal y como Trimborn (1949:281) acertadamente reconoce. Aunque Carneiro (1991:176) ha presentado esta misma cita más recientemente y esta vez sí en la manera debida, no reconoce su error anterior. Lo que es importante resaltar aquí para nuestra discusión es el uso de citas en esta forma, con el ánimo de perpetuar y hacer énfasis en unas condiciones como generales, cuando estas se refieren a casos específicos. En este sentido se debe aclarar que el trabajo de Trimborn (1949), el cual es casi la única fuente que Carneiro $(1990,1991)$ utiliza para sus argumentos, requiere una lectura muy atenta, toda vez que frecuentemente recurre a la información de grupos fuera del valle del Cauca para ampliar sus observaciones, lo que genera problemas adicionales. Son repetidos los casos en que las generalizaciones u observaciones que Trimborn presenta, parecen más palabras de un 'testigo ocular' que una reconstrucción hipotética (véase por ejemplo, la cita traducida por Carneiro (1990:193) sobre la naturaleza de la guerra en el valle del Cauca). Así que, si bien en principio estoy de acuerdo con las observaciones que Carneiro $(1990: 193 ; 1991$ : 169) hace sobre las virtudes académicas de Trimborn, creo, no obstante, necesario llamar la atención sobre estos aspectos.

7 Se debe hacer mención especial del estudio de Schorr (1965) el cual es, sin lugar a dudas, un intento claro e interesante de análisis interdisciplinario de los patrones de asentamiento en el valle del Cauca. 
conflictos intertribales ${ }^{8}$. Por el contrario, las batallas que dan pie y sirven de base para la idea generalizada de guerras genocidas crónicas, fueron no sólo motivadas por la presencia de los españoles sino, además, por guerras en las que ellos mismos tuvieron un papel protagónico (Cieza 1985a:CXXII:34). Para evaluar ésto con mayores detalles, vale la pena mirar brevemente la secuencia de eventos de la campaña española en la región del valle medio del Cauca.

Cuando el capitán Jorge Robledo cruzó el río Cauca desde la provincia de Anserma hasta la de Carrapa, ya había él concluido la primera campaña de "pacificación" (Sarmiento [1540] 1864:279). A su arribo, después de una demostración inicial del poderío español (Sarmiento [1540] 1864:281), Robledo pudo organizar un ejército de alrededor de 4.000 guerreros y 600 cargadores Carrapas (Cieza 1985b:C-VII:166), los cuales se unieron a los españoles, Ansermas, esclavos africanos y yanaconas que viajaban con él, para así internarse en el territorio de los Picaras. El resultado de esta campaña fue la matanza y posterior canibalismo de un número alto de víctimas (Cieza 1985b:C-VII:166).

La próxima campaña, contra los Pozos, contó con la participación de los Picaras, quienes proporcionaron una fuerza superior a los 4.000 guerreros (Cieza 1985a:C-XXII:34). En otro pasaje, no obstante, Cieza (1985 1985b:CVII: 167) anota que eran más de 5.000 e indica también que “...había más de diez o doce mil indios de guerra cuando la primera vez entramos en esta provincia" (Cieza 1985a:C-XXII:33). Cualquiera que hubiese sido el número exacto de guerreros, lo que parece claro es que el ejército español alcanzó un tamaño aproximado de 8.000 soldados (Cieza 1985b:C-VIII:167), que se enfrentaron a un ejército Pozo de unos 6.000 guerreros (Cieza 1985b:C-VII:167). En contraste, Sarmiento ([1540] 1864:282) anota que el ejército Pozo era de más de 4.000 personas. El resultado de esta campaña fue la muerte de un alto número de Pozos. En un episodio particular, en el cual alrededor de 1.000 personas trataban de escapar a lo alto de una loma, 300 de éstas perecieron. La acción proporcionó a los Picaras y Carrapas, 200 cargas de carne aproximadamente; parte de ésta fue consumida en el campo de batalla y el remanente enviado a sus respectivos lugares de origen (Cieza 1985b:C-IX:168).

8 La otra región considerada por Carneiro es la de Fiji. Aunque aquí no se discutirá, vale la pena anotar, eso si, que a partir del texto parece claro que las descripciones de esta zona podrian tomarse literalmente como "observaciones neutrales". Vale la pena tomar en cuenta en este sentido el sumario presentado por Ferguson (1990:51-54) sobre los problemas que genera el desconocimiento de los efectos que la presencia de los grupos occidentales tiene en la transformación de los patrones de guerra indigenas. 
Posteriormente, Robledo organizó la campaña contra los Paucuras, en la que los Pozos proporcionaron 'muchos' indígenas para cargar las provisiones (Cieza 1985b:C-IX:169). Los Paucuras, conocedores de lo que había sucedido en la provincia de los Pozos, no opusieron resistencia y por el contrario, los recibieron con "...mucho mantenimiento aparejado..."(Cieza 1985b:C-X:169). Una vez en Paucura, Robledo consiguió la ayuda de alrededor de 3.000 guerreros para emprender otra expedición contra los Pozos, en teoría, con el propósito de castigarlos por el robo de unos cerdos. Cieza 1985b:C-X:169) declara que en tal ocasión, los Paucuras mataron más de 200 Pozos cuyos cuerpos trajeron de regreso a su territorio para comérselos. Debe anotarse aquí, no obstante, que la verdadera razón de esta segunda campaña contra los Pozos fue muy diferente. Cieza 1985b:CIX:168) comenta que, puesto que Robledo había sido severamente herido en el curso de la primera campaña contra los Pozos, los españoles decidieron vengar tal hecho al punto que "...llevaban los que iban a hacer el castigo voluntad de no perdonar la vida a ninguno".

La siguiente campaña fue contra los Armas y en ésta los españoles contaron con la ayuda de 'muchos' Paucuras. Cieza (1985a:C-XVIII:29), quien anota que los Armas tenían más de 20.000 guerreros, no establece que se haya practicado canibalismo con los cuerpos de las víctimas de esta campaña. La resistencia ofrecida por los Armas fue, no obstante, fiera. Cieza (1985a:C-XI:171) anota que,

"En este tiempo, los naturales de la provincia de Arma habían cobrado tanto odio a los españoles, que generalmente determinaron de darles guerra, y así lo pusieron por obra,... Y... el capitán determinó de salirse de la provincia, dejándola tan de guẹrra como al principio que en ella entramos;"”.

De los anteriores eventos se han derivado dos conclusiones principales. La primera, que estos grupos participaron voluntariamente como aliados de los españoles, quienes perseguían de esta manera sus propios fines políticos o militares. La segunda, que la crueldad (i.e, el gran número de víctimas) así como el tamaño de los ejércitos indígenas, reflejan directamente las condiciones imperantes en tiempos prehispánicos. Para evaluar estas proposiciones es necesario considerar en más detalle algunos hechos.

En primer lugar, se debe anotar que para el momento en que los españoles llegaron a estas tierras, los indígenas ya sabían del significado de la 'paz' española, así como del costo de mantener su independencia. Que esto ocurrió de esta manera puede verse en la siguiente cita tomada de 
Cieza (1985b:C-VII:166) en la que describe el arribo a la provincia de Carrapa,

“... como en todas aquellas comarcas se hubiese ya dicho del valor de los españoles y de su mucho esfuerzo, y de la fortaleza de sus caballos, acordaron, por no verse heridos con sus espadas y despedazados con los perros, de acogerlos en su provincia y proveerles de bastimentos..."

Así las cosas, la opción política de estos grupos era la de resistir o la de convertirse en aliados de los españoles. Si una constante puede notarse entre todos estos grupos, esta sería la de un estado inicial de confrontación o resistencia y luego el establecimiento de alianzas con los españoles, las cuales eran de una naturaleza débil. El objetivo de tales alianzas era asegurarse que los españoles se retiraran de sus territorios a otras tierras más 'ricas'. Cieza (1985b:C-XV:176) anota que los Quimbayas “...no solamente no se pusieron en resistencia, mas determinaron de salir al capitán ....con presentes de oro, creyendo que pasarían de largo;...". Tan pronto como los indigenas se dieron cuenta que ese no sería el caso, las rebeliones comenzaron. Cieza (1985a:C-XXIV:36) aun anota que la pacificación de estos grupos sólo fue exitosa "...hasta que se hizo justicia [léase ejecución] de los caciques antiguos”.

Al evaluar la idea que estos grupos participaron en estas campañas movidos por el fin de promover sus propias agendas politicas, hay al menos dos hechos que llaman la atención. El primero, que estos grupos no recibieran en compensación los territorios de los grupos contra los cuales pelearon (o al menos parte de estos), sino víctimas para canibalismo y en algunos casos, algunos prisioneros, cuya suerte, de acuerdo con los datos, fue seguramente el sacrificio.

El segundo hecho interesante en relación con la naturaleza de estas alianzas es, que de acuerdo con las crónicas, los grupos que se encontraban en conflicto al momento de la llegada de los españoles, participaron conjuntamente en campañas contra otros grupos. Tal es el caso, por ejemplo, de los Carrapas y Picaras, quienes marcharon conjuntamente contra los Pozos (Cieza 1985b:C-VIII:167).

Los episodios de resistencia indígena, incluyendo aquellos que se originaron después de la primera campaña española de 'pacificación' (véase Friede 1982:53, 77-93; Valencia 1991:43-56; Cieza 1985b:C-X:171; CCIX:407-409, C-CXLIII:447-450, C-CLIX:469-470), sirven también para 
ilustrar la naturaleza temporal de estas alianzas, así como para sustentar la noción que éstas se encaminaban a propiciar la salida de los españoles de los territorios de cada grupo o de la región como una totalidad.

En relación con la idea que la guerra en tiempos prehispánicos se caracterizó por altos niveles de 'crueldad' y un índice alto de mortalidad, así como de ser llevada a cabo con ejércitos de considerable tamaño, varios argumentos deben ser considerados. Comencemos por ver el aspecto del tamaño de los ejércitos, el cual ha sido utilizado como un indicador general del estado endémico de guerra entre estas comunidades (cf. Carneiro 1990:195-196; Trimborn 1949:336).

La pregunta central que nos debemos formular en este sentido es si estos ejércitos son un reflejo directo del tamaño de los ejércitos que cada grupo podía conformar en cualquier momento, proveyéndonos así de un sólido indicador para inferir niveles de organización sociopolítica anteriores a la conquista. El punto a considerar aquí es, que la guerra requiere organización e implica la presencia de estructuras complejas para hacer posible la consolidación de ejércitos de tales magnitudes (ver Turney-High $1971: 227,228)$.

Todas las referencias indican, no obstante, que entre los Carrapas, Picaras, Pozos y Paucuras, por ejemplo, el poder para llamar y conformar ejércitos de esas magnitudes no estaba en las manos de un solo individuo, sino que dependia del consenso entre múltiples niveles de poder de orden local $^{9}$. De acuerdo con el argumento aquí propuesto, este hecho es más consistente con la idea que la habilidad para constituir tales ejércitos fue una respuesta y consecuencia-directa de la presencia española.

Es interesante anotar en este sentido que Carneiro (1990:196) estima el tamaño promedio de los ejércitos de estos grupos entre 200 y 400 guerreros. Tomando en consideración las dificultades para estimar tanto la

9 Información sobre este tópico se encuentra en Cieza ([1550] 1985: T-I,C-XIX:31) para los Pozo, en Cieza ([1550] 1985:T-I,C-XXIII:34) para los Paucura, en Cieza ([1550] 1985:T-I,C-XXII:33) para los Picara y en Cieza ([1550] 1985: T-I,C-XXIII:35) para los Carrapa y compare estos con Cieza ([1550] 1985:T-II,C-V: 163) para el caso de Anserma. El punto de interés en este sentido es, que si nos ceñimos a los criterios establecidos por Carneiro (1991:168) para definir una sociedad de tipo cacical, los grupos anteriores tendrian que ser excluidos como tales por las mismas razones que él plantea para excluir aquellos de la Costa Noroeste de los Estados Unidos. 
organización sociopolítica de estos grupos (es decir, cuantas tribus o cacicazgos, el tamaño de las poblaciones, etc, [ver Trimborn 1949:334 para una discusión de estos aspectos]), la magnitud de la diferencia entre el promedio anotado por Carneiro y el tamaño de los ejércitos citados por las crónicas (entre 4.000 y 20.000) habla claramente de lo excepcional de estos grandes ejércitos, asi como de las condiciones específicas en que se conformaron ${ }^{10}$.

El punto a destacar aquí es que si los ejércitos de gran tamaño fueron la norma (y no la excepción), bien se podría esperar encontrar en los documentos de la época una descripción diferente de esta región en términos de organización sociopolítica o, cuando menos, se esperaría encontrar referencias frecuentes que apuntaran a cambios en tal sentido, tales como la incorporación de unos grupos a otros y/o la expansión del territorio de unos grupos a expensas del de otros. El hecho claro, es sin embargo, que aunque los españoles se interesaron por indagar sobre la historia de estos grupos (ver Cieza 1985a:C-XXI:31; C-XXIII:35, C-XXIV:36), las referencias en tal sentido son muy pocas. Uno de las casos más claros es el de los Quimbayas, de quienes se dice exterminaron a los antiguos habitantes del territorio que entonces ocupaban. No obstante, este evento dramático, no ha sido confirmado desde una perspectiva arqueológica.

En el mismo sentido, si nos concentramos en el tamaño de la fuerza combinada de Carrapas, Picaras y Paucuras, todos éstos descritos como 'enemigos naturales' de los Pozos, se podría esperar que tal grupo hubiese sido exterminado antes de llegar los españoles, asumiendo que el estado de guerra que prevalecía en la región era, en verdad, uno de "guerra de todos contra todos, llevada a cabo mediante todo posible medio coercitivo, militar y no militar" (Carneiro 1990:193 citando a Trimborn 1949).

Cieza (1985a:C-XXI:31; 1985b:C-VIII:167), quien sistemáticamente describió a los Pozos como los más fieros de todos los guerreros en la región, anota que considerados por aparte los Carrapas, Paucuras y Picaras tenían una población que en cada caso triplicaba la de los Pozos (Cieza 1985a:C-XXI:32). En tales circunstancias, uno podría esperar que como resultado de la acción de guerra de estos grupos contra los Pozos, este último hubiese sido exterminado o su población reducida drásticamente. Que esto no haya sido lo que los espa-

10 Se debe indicar que Carneiro (1991:177) presenta la información sobre el tamaño de los ejércitos sin indicar o aclarar en ningún momento que estos ejércitos se constituyeron bajo el mando y motivados por la presencia española. 
ñoles encontraron, puede tomarse como punto de partida para cuestionar una vez más no sólo el carácter extraordinario de los grandes ejércitos indígenas ya discutidos, sino también para preguntarnos qué tan diferentes pueden haber sido los patrones de guerra en tiempos verdaderamente prehispánicos. Esto, de hecho, pone también en tela de juicio los argumentos que investigadores modernos han propuesto al tomar como punto de partida las observaciones generales de los cronistas.

Finalmente, debemos considerar que, si la guerra entre estos grupos fue en verdad un hecho crónico (total y sin fin [Carneiro: 1990:199]), si las matanzas fueron indiscriminadas (i.e, incluyendo hombres, mujeres y niños por igual), y lo más importante, si la guerra era practicada por ejércitos numerosos, se podría esperar, en principio, la presencia de una población regional baja como consecuencia directa de una relación negativa y sostenida, entre las ratas de reproducción y muerte (tomando como parámetro el número de víctimas resultantes de los conflictos descritos [véase Carneiro 1990:199 y compárese con Gregor 1990:124]).

Nos parece interesante anotar que los españoles, a pesar de las repetidas referencias en las cuales estos grupos son declarados 'enemigos naturales' desde antes de su llegada, nunca establecen que los niveles de población observados por ellos reflejen la acción negativa (baja) de la guerra. Comentarios sobre el decrecimiento de la población aparecen sólo a partir del momento en que los españoles comienzan a comparar lo que sucede después de su entrada. En tales circunstancias, no obstante, la reducción de población se explica como resultado de la escasez de alimentos y por la acción de diversas epidemias, más que como efecto de los combates o la guerra en sí misma. La escasez de alimentos, por su parte, se presenta como un resultado de la negativa de los indígenas a cultivar los campos, para hacer la supervivencia de los españoles más difícil; en otras palabras, como una estrategia militar para propiciar la retirada de los españoles de sus territorios (véase por ejemplo Cieza 1985a:CXXX:44).

Algunos investigadores (cf. Trimborn 1949:280-295; Carneiro 1991:177), también han argumentado que la destrucción de las aldeas y cultivos son elementos que demuestran que la guerra entre los grupos del valle del Cauca era de una naturaleza total, sin fin y de exterminio. A pesar de esto, es fácil ver que tales prácticas, aunque fueron usadas tanto por los indígenas como por los españoles, no parecen haber sido la norma o patrón de las guerras entre estos grupos. La siguiente cita de Cieza (1985b:C-CXLIII:448) es de particular interés en este sentido. El cronista anota que puesto que los Picaras estaban 
resueltos a resistir la presencia española a como diera lugar, los españoles decidieron destruir sus cultivos para así forzarlos a rendirse. Pero los Picaras, quienes usaban antorchas para cultivar de noche, reclamaban de los españoles que,

" la guerra la continuasen todo el tiempo que ellos quisiesen, y que no destruyesen los mantenimientos, sino que comiendo a discreción de ellos, dejasen los demás para que ellos hiciesen lo mismo, pues ellos los sembraban".

Esta restricción en las prácticas de guerra no es un rasgo exclusivo de este grupo en particular, sino un rasgo bastante extendido (cf. Blick 1988:658-659, 662-663). La presencia de estas restricciones, parece, por lo tanto, más consistente con el argumento aquí propuesto, que con aquéllas basadas en la aceptación literal de las descripciones de los conflictos entre indigenas y españoles proporcionadas por los cronistas.

Otro elemento relacionado con la naturaleza de la guerra, y que requiere consideración, es el de los sistemas defensivos. Carneiro (1990:197), probablemente siguiendo a Trimborn (1949:120), anota que "las villas estaban generalmente defendidas con sistemas de palizadas hechas de guaduas". Con todo, este argumento parece estar en contradicción con las descripciones proporcionadas por los españoles mismos. Ellos reconocen la presencia de palizadas, pero siempre localizadas al frente de las puertas de las casas de los caciques y otros individuos de importancia; las cuales, si bien en ocasiones fueron usadas como sistemas defensivos, parecen haber servido principalmente como epicentro de actividades ceremoniales y rituales (cf. Robledo [sf.] 1865:401-402; Cieza 1985a:C-XVI:26; C-XIX:29; C-XXI:32; C-XXII:33). Cieza (1985a:C-XXII:33; C-XV:25) describe estas estructuras para el caso de los Picaras y Ansermas, de la siguiente manera, respectivamente:

"A las puertas de las casas de los caciques hay plazas pequeñas, todas cercadas de las cañas gordas, en lo alto de las cuales tienen colgadas las cabezas de los enemigos...Por lo bajo de las cañas hacen unos agujeros por donde el aire puede respirar cuando algún viento se levanta; hace gran sonido, parece música de diablos."

"Los señores o caciques y sus capitanes tienen casas muy grandes, y en las puertas de ellas puestas unas cañas gordas....encima de ellas tienen puestas muchas cabezas de sus enemigos... Entre estas cañas tienen puestas unas tablas, donde esculpen la figura del demonio, muy fiera, de manera humana, y otros ídolos y figuras de gatos, en quien adoran... A las puertas de las casas que he dicho tienen plazas pequeñas, sobre las cuales están puestas las cañas gordas; y en estas plazas tienen sus mortuorios y sepulturas al uso de su patria,...". 
Un segundo elemento para considerar en este sentido es, que la información sobre patrones de asentamiento indica que existía gran variabilidad en cuanto a la estructura o disposición de los asentamientos, incluyendo tanto habitaciones comunales y unidades domésticas nucleares, así como asentamientos dispersos hasta más o menos compactos (ver Trimborn 1949:125-133 para una discusión) ${ }^{11}$. El punto importante aquí es cómo la ausencia de asentamientos verdaderamente compactos $\mathrm{y}$, por ende, la ausencia de aldeas verdaderamente rodeadas por palizadas, apunta hacia una situación (al menos) diferente de aquella que la cita de Carneiro indica.

La presencia de cabezas humanas-trofeos y momias en el interior ó alrededor de las casas de los caciques y principales, también ha sido usada por algunos investigadores para reforzar su argumento de la escala de belicosidad de estos grupos. Los problemas asociados con la interpretación de tales elementos como prueba de los niveles de guerra entre estas comunidades son varios. En primer lugar, es necesario considerar que las cabezas trofeos operaban en un contexto simbólico y ritual mediante el cual los caciques y principales reforzaban sus posiciones de prestigio, como toda la evidencia parece indicar (cf. Cieza 1985a:C-XXVI:39; Carneiro 1990:201); por lo tanto, es de tener en cuenta que tales cabezas no representan el producto de eventos particulares sino el producto de un proceso acumulativo cuya duración se desconoce.

Como segundo hecho para considerar en relación con estos símbolos figura el que ellos no aparezcan descritos entre los objetos que fueron sistemáticamente enterrados con el cuerpo de los caciques (ver Robledo [sf.] 1865:396; Cieza 1985a: C-XVI:25, C-XV:27). Esta situación deja abierta la posibilidad que tales símbolos fuesen heredados por el sucesor (ver Trimborn 1949:224 y Helms 1980:721-722, 1976 sobre sucesión) como parte de la herencia del cacique. En tal caso, la cantidad de cabezas-trofeo no puede ser la base para afirmaciones sobre la intensidad de la guerra o sobre el grado de belicosidad.

1 Las afirmaciones de Trimborn en materia de patrones de asentamiento son a veces contradictorias. Así, éste comienza la discusión de este aspecto diciendo que, "El hecho de que los poblados predominaran en forma de aldeas y no de chozas dispersas se refleja con tal regularidad en las descripciones de los testigos oculares que hace innecesaria su demostración documentada" (1949:125). Posteriormente, no obstante, anota que, "Es de suponer que entonces predominara el tipo de aldea de planta irregular. Pero había partes en que la colonización mostraba un tipo disperso, sin que por ello hubiera faltado una comunidad local" (1949:129). Véase también Ford (1944:13) y Schorr (1965:77) para una discusión sobre patrones de asentamiento entre los Gorrones. 
Una pieza de evidencia relacionada con este problema y frecuentemente citada como prueba de la crueldad de las gentes del valle del Cauca es el reporte de una casa cerca a Cali, en la cual había una gran cantidad de momias que eran mantenidas para celebrar la ferocidad del cacique local (Cieza 1985a:C-XXVIII:41). Andagoya (citado en Jijón y Caamaño 1938: Documento $3: 56$ ) anota que en esa casa había " 400 hombres o lo que cabían en aquella sala". Dejando de lado la duda de cuántas había realmente, el punto a considerar es lo muy común que la práctica de la momificación era en la región (cf. Robledo [sf.] 1865:396; Cieza 1985a:C-XVI:27) ${ }^{12}$. Un reporte anónimo (1886:489) anota que " A unos entierran debajo tierra, a otros ponen al humo, donde se seca, y así seco lo guardan."

Refiriéndose al territorio de los Cueva, Oviedo (1853:455) observa que en algunos lugares, las momias de los caciques se guardaban en una casa y que el orden en el cual estaban colocadas servía para proporcionar información sobre la genealogía y parentesco, así como sobre el número de caciques que ese estado había tenido. Aunque no podemos asegurar que esta fuese la situación en el caso reportado para Cali, ello nos sirve para ilustrar los problemas que se enfrentan al usar tales evidencias para sustentar afirmaciones sobre el grado de guerra y canibalismo. Como Pineda (1987) lo ha discutido ya, este podría ser un buen ejemplo sobre manipulación de información con fines políticos.

La contemporaneidad de los reportes españoles en relación con estas dos clases de símbolos (momias y cabezas-trofeo) así como el ambiente no aborigen de las condiciones en las cuales se desarrollaban las guerras narradas por los cronistas, son otros elementos para tener en cuenta en cualquier intento de reconstrucción de los patrones y características de la guerra en tiempos prehispánicos. Tal y como se ha argumentado en este articulo, la totalidad de los eventos políticos y militares descritos en las crónicas son hechos que gravitaban alrededor de la presencia activa de los españoles en el ámbito de los acontecimientos. Debemos hacer énfasis aquí en que las únicas referencias que traen las crónicas sobre eventos de guerra de auténtico carácter aborigen o prehispánico, son hechos que tuvieron lugar mucho antes del arribo de los españoles o que, en su defecto, fueron "congelados" como consecuencia de su llegada ${ }^{13}$. En cualquier caso, estos hechos no fueron "observados" por los

12 Para una discusión sobre la manipulación política de momias véase Langebaek (1992) y Zudeima (1983)

13 La excepción, en lo que concierne al área de estudio en este trabajo, son los casos ya mencionados sobre los Carrapas y Picaras (Cieza [1550] 1985:T-I,C-XXI:31) y el caso de Irrua (Cieza [1550] 1985:T-I,C-XXIII:35). 
españoles. Con relación al caso de los Quimbayas, mencionado antes, Cieza (1985a:C-XXIV:36) comenta que,

"También antiguamente no eran naturales estos indios de Quimbaya, pero muchos tiempos ha que se entraron en la provincia matando a todos los naturales, que no debían ser pocos, según lo dan a entender las muchas labranzas, pues todos aquellos bravos cañaverales parecen haber sido poblado y labrado, y lo mismo las partes donde monte hay: que hay árboles tan gruesos como dos bueyes, y otros más. Donde se ve que solia ser poblado, por donde yo conjeturo haber gran curso de tiempo que estos indios poblaron en estas Indias. El temple de la provincia es muy sano, adonde los españoles viven mucho y con pocas enfermedades, ni con frío ni con calor".

Aunque el argumento expuesto por el cronista en este pasaje es, sin lugar a dudas, interesante, es necesario resaltar que esas evidencias pueden ser el resultado de otros procesos o fenómenos. Por ejemplo, la presencia de los campos abandonados puede estar relacionada con las prácticas agrícolas de estos grupos (cada cuánto se abandonaban las parcelas para regeneración ?) y de las que no tenemos mucha información al respecto ${ }^{14}$. Desde otra perspectiva, cabe anotar que un evento de la escala que representa el exterminio de un grupo y la subsecuente ocupación del mismo territorio por otro grupo - evento que en principio es posible corroborar mediante la investigación arqueológica (véase por ejemplo Balée 1984:249) - , no ha sido hasta el momento confirmado. Arocha (1987:34) sin embargo, afirma que tal evento ocurrió en el siglo XVI.

Tomando en consideración los problemas discutidos hasta aquí, debemos enfocarnos ahora en las condiciones estructurales que según la opinión de investigadores modernos, determinaban tanto el canibalismo como la guerra, para explorar asi otras dimensiones de este problema.

\section{CONDICIONES ESTRUCTURALES DE LA GUERRA Y EL CANIBALISMO}

Como se indicó antes, existen dos argumentos principales para explicar la guerra en el valle del Cauca. El primero de éstos (cf. Arocha 1987) ve la guerra como un mecanismo encaminado a conseguir víctimas para canibalismo alimenticio tendiente a subsanar las deficiencias en el suministro de proteínas,

14 En la zona Calima, por ejemplo, los cambios de temperatura y humedad al parecer causaron el abandono de camellones (Herrera et al. 1990). 
causadas por la presión demográfica sobre los recursos naturales (para un resumen sobre el debate de las "proteinas" véase Ferguson 1984:31-37). El segundo argumento, por su parte, ve la guerra emergiendo de la necesidad de proporcionar victimas para canibalismo ritual en el contexto de ceremonias mágico-religiosas (Carneiro 1990). A continuación exploraremos estos argumentos con mayor detalle ${ }^{15}$.

Arocha (1987:32-34) argumenta que la guerra, al proporcionar las víctimas para un canibalismo alimenticio, tenía tal papel debido a la alta densidad de población y a la dificultad para la expansión territorial, dada la compleja topografia de la región, y la que bien se puede denominar 'circunscripción social' (para usar la terminología de Carneiro 1970, 1981, 1990) reinante en la zona.

Mirando la situación desde una perspectiva regional, uno bien podría asumir que la circunscripción territorial fue en realidad un hecho claro debido al gran número de grupos que habitaban la región, de acuerdo con las crónicas, pero sobre todo, por la aparente proximidad territorial entre estos grupos. Robledo ([sf.] 1865:400) por ejemplo, señala que los campos de cultivo de los Pozos y Picaras estaban juntos. Cieza (1985a:C-XXI:32), no obstante, afirma que la provincia de los Picaras estaba a unas dos leguas de distancia de la de los Pozos, mientras que la de los Picaras y Paucuras estaba a legua y media de distancia.

Asi y todo, esta proximidad entre grupos se basa en los limites territoriales de acuerdo con un eje norte-sur. Pero, qué del límite oriental? La falta de información en este sentido se debe no sólo a las dificultades que los españoles encontraron cuando trataron de explorar esa zona debido a la vegetación densa y a la topografía quebrada de la zona, sino también como resultado de la resistencia directa opuesta por los indígenas, en unos casos, y temida, en otros (Sardella [sf.] 1864:307). Si los habitantes de esa zona eran o no, parte de las mismos grupos aquí discutidos, es algo que no sabemos con seguridad.

La implicación más directa que ésto tiene en el caso que fuesen gentes de los mismos grupos las que poblaban las zonas hacia la parte alta de la

15 Al hacer la siguiente discusión, retomo la posición expresada por Chagnon (1990:89) en torno a la discusión sobre proteinas y en la que considera que aunque la información confiable ("hard data") es tan escasa y dificil de acumular, es válido asumir que en muchos casos, la presencia de reiteradas referencias sobre el mismo hecho así como la sensación de coherencia entre éstas, puede ser tomada como base confiable para una discusión. 
cordillera Central, es que este factor se convertiria en una variable relevante para cualquier intento de determinar la presencia de una presión demográfica (i.e, de la relación existente entre densidad de población y recursos) y, por lo tanto, en cualquier evaluación que quiera explicar la guerra como mecanismo de adquisición de víctimas para canibalismo con fines nutricionales. La inclusión de esas áreas, elevaria la cantidad de tierra y el número de recursos disponibles ${ }^{16}$.

Con relación a la disponibilidad de tierra y alimentos, debemos señalar que la mayoría de los documentos españoles establecen claramente que ambos recursos existian en abundancia. Cieza (1985a:C-XIII:23), al hacer un paralelo entre la región del valle del Cauca y la costa peruana, puntualiza que en la primera, cuando los indigenas eran perseguidos por los españoles, se desplazaban a otra zona, construían casas y en cuatro meses comenzaban a cosechar de nuevo porque "... adonde quiera que van o están, hallan qué comer y tierra fértil y aparejada y dispuesta para darles fruto". Los indígenas del Perú, por su parte, no podian hacer lo mismo porque las áreas de suelos fértiles eran limitadas y se encontraban separadas entre sí por extensas zonas desérticas. La expedición de Robledo a lo largo del río Cauca también indica la presencia de extensas zonas deshabitadas o no cultivadas (ver Sardella [sf.] 1864:295). En la zona del Quindío se describen también extensas zonas de bosque (Sardella [sf.] 1864:303). Estas zonas 'vacias' pueden haber servido como zonas limitrofes o zonas de amortiguamiento entre grupos diferentes, en las que ambos podían cazar u obtener otros recursos.

Los problemas relacionados con el argumento de una presión demográfica sobre los recursos en esta zona pueden ilustrarse también a nivel micro-regional con el argumento de Arocha (1987:34) sobre los Quimbayas. Este anota que la densidad de la población era de alrededor de 10-11 habitantes por kilómetro cuadrado, pero puesto que toda la ocupación estaba restringida a las alturas medias, tal figura puede haber alcanzado los 60 habitantes por kilómetro cuadrado. Con esta base, él concluye que por estar la mayoria de los cultivos adaptados a la zona media, se originaba una situación de zonas con potencial agrícola limitado, y de conflicto por el control de tales áreas (1987:34).

16 La evidencia arqueológica disponible sobre estas zonas del extremo Este, alrededor de la zona de Manizales (véase Jaramillo 1991), indica la presencia de una ocupación relativamente densa. En lo que hace relación a los asentamientos Quimbayas desde el punto de vista ecológico, véase Friede (1963:307) y Schorr (1965:105). A nivel arqueológico en general, véase Salgado (1986), Cubillos (1984), Castillo (1988), Brunhs (1981) 
A pesar de todo, este escenario contrasta de varias maneras con las descripciones de los cronistas. Cieza (1985b:C-XV:176) anota la presencia de extensos bosques de "cañas gordas' (guaduas) en el contexto de las elevaciones medias de la zona Quimbaya, zonas tan extensas y densas que cuando los españoles comenzaron la exploración de esta región, muchos soldados se resistieron a seguir adelante, puesto que no podían ver en la distancia ni los valles ni los asentamientos indigenas tal y como los veían en las zonas norteñas. Sin embargo, los valles y asentamientos estaban allí, sólo que ocultos por los bosques de guadua. Robledo ([sf.] 1865:398) describe el territorio Quimbaya de la siguiente manera:

“...la tierra es más llana que la de Santa Ana [Anserma], sino que es toda llena de cañaverales y entre ellos tienen los indios hechos sus pueblos, porque descienden desdesta sierra nevada muchos ríos pequeños, que van á dar a este río [Cauca] é pasan por estas provincias donde se hacen muy grandes vegas é ríos, donde los indios tienen sus sementeras é grangerias é grandes arboledas de frutas. Hay entre estos cañaverales mucho género de caza, porque aunque hay cañas, no es tierra de anegadizos, ni hay ciénagas, sino tierra buena y muy sana."

La situación de desequilibrio entre densidad de población y recursos que Arocha propone, también contrasta con reportes recurrentes sobre la naturaleza de los recursos alimenticios, los cuales establecen con claridad que éstos eran abundantes y diversos (cf. Cieza:1985a:C-XVII:27; CXIX:30; C-XXIV:35; C-XXV:37; C-XXVI Y XXVII:40; 1985b:C-XI:170; C-XV:177; Sardella [sf.] 1864:294). Adicionalmente, según estas mismas fuentes, es claro que la estación de cultivo no estaba restringida a unos pocos meses del año (Robledo [sf.] 1865:395; Cieza 1985a:C-XVII:28; ver también Tovar 1992: 60-63 sobre el tema de la diponibilidad y variedad de recursos).

Dadas estas consideraciones, tenemos que el argumento sobre la deficiencia de proteínas animales resulta, al menos, difícil de sostener. Arocha ha argumentado que en el valle del Cauca sólo los Gorrones tenían un eficiente suministro de proteína animal (o, al menos, un suministro substancial). Este argumento se basa en la descripción de un pueblo indígena a la orilla del río Cauca en el cual había una laguna (si era natural o artificial no se establece) de la que se obtenían grandes cantidades de pescado; el pescado, una vez ahumado o secado al fuego, se utilizaba para comercio con otras regiones (Sardella [sf.] 1864:293). La presencia de vasijas grandes para almacenar aceite de pescado fue reportada también en 
este lugar (Cieza 1985a:C-XXVI:40) ${ }^{17}$. El punto a considerar aquí es, que este sistema excepcional para la explotación de pescado (el cual probablemente resultaba de la combinación de las características bióticas propias del río Cauca [véase Schorr 1965:90] con el sistema de estanques o lagunas naturales) no puede ser tomado como garantía para establecer un argumento de distribución 'eficiente' de proteínas entre el grupo Gorron y tampoco tiene paralelo en el resto de la zona. Asi mismo, la existencia de un comercio de pescado no puede ser tomado como prueba de una situación extrema de falta de proteínas animales en el resto de la región.

En cuanto a la distribución 'eficiente', debemos anotar que no existe información sobre cómo se beneficiaban los Gorrones de esta producción. Por ejemplo, tenían todos acceso a la misma cantidad, o los caciques tenían una mayor participación? En cuanto a la disponibilidad de proteína animal en el resto de la zona, sabemos que, tal y como es evidente en la cita de Robledo mencionada arriba, la zona estaba cruzada por una gran cantidad de ríos en los que se practicaba la pesca. Friede (1982:112-115), por ejemplo, ha documentado que entre los Quimbayas, aún en el año de 1559, con la excepción de cuatro encomiendas, todas las demás tributaban pescado, además de otra serie de productos. Se podría argumentar que si las condiciones de disponibilidad de proteínas animales eran tan extremas en el resto de la zona, localidades como el pueblo descrito en el territorio Gorron deberian haber sido un rasgo común en las márgenes del río Cauca, al menos en la parte entre Cali y el comienzo del cañón del río Cauca. En ésta extensión considerable de territorio sin lugar a dudas, se habrían podido llevar a cabo prácticas piscícolas como la de los Gorrones.

En relación con la disponibilidad de alimentos cabe observar que en las referencias sobre comercio (con excepción del caso de los Gorrones y el de la sal) no se menciona la circulación de alimentos pero sí de productos tales como oro, algodón y tejidos (cf. Cieza 1985a:C-XVII:28; Anónimo 1886:492).

La función del canibalismo como fuente de proteínas, también contrasta con las referencias que los cronistas hacen sobre esta práctica, las cuales indican modestos niveles de canibalismo dejando de lado, claro está, aquellas que se originan en el contexto de conflictos con la participación

17 En relación con este sistema de explotación de pescado, ver también Tovar (1992:60), en especial la nota 81 donde analiza el aspecto de medidas y volumenes. 
activa de españoles tal y como ya se ha discutido (véase Cieza 1985a:CXX:31, pero compare con Cieza 1985a:C-XXI:32-33, donde como resultado de un combate en el que los españoles participan se reportan 100 individuos comidos). Robledo ([sf.] 1865:393) anota que en Anserma,

"Los indios ... comen muy poca carne; lo más de su comer es frutas e yerbas guisadas de muchas maneras, con axi y comen muy poca carne humana y la que comen es de indios de guerra de tierras lejas, la carne que comen es de caza, porque hay mucha, hay muchos géneros de frutas muy buenas" 18

La referencia anterior efectivamente ilustra el hecho que el acceso a proteínas animales podía derivarse de una amplia gama de especies además del pescado.

Entre los Quimbayas, la naturaleza ritual y no alimenticia del canibalismo también parece clara. Cieza (1985a:C-XXIV:35) comenta que "No comen carne humana sino es por muy grande fiesta". Para el caso de los Armas y Paucuras, Cieza (1985a:C-XX:31) anota que:

“..cada martes sacrificaban dos indios al demonio ....según nos dijeron los indios; aunque éstos que sacrificaban, si lo hacían, tampoco alcanzo si serían de los mismos naturales o de los que prendían en la guerra."

El escepticismo de este cronista sobre la práctica del canibalismo en el caso particular, tomando en consideración que él mismo sistemáticamente describe estos grupos como caníbales crónicos, amerita algunas observaciones adicionales. Se podría tomar esta misma referencia como punto de partida para argumentar qué tan poco frecuentes o qué tan excepcionales eran aquellos actos masivos de canibalismo de que hablan los cronistas. Pero es probablemente una referencia acerca de los mismos Gorrones la que resulta más contradictoria para el argumento de las proteínas, puesto que si su función era la de suplir proteínas - y los Gorrones no tenían este problema- el canibalismo no tendría razón de existencia entre ellos. Cieza (1985a:C-XXVI:39) comenta en este sentido que:

is Esta cita muestra claramente la ambigüedad de los textos. Robledo comienza por establecer que los indigenas comen muy poca carne; luego anota que la fauna era abundante. Entonces uno puede concluir que si el consumo de carne era bajo, ésto se debía a razones culturales y no a la falta de animales de caza. Excavaciones de basureros y áreas de cocina podrian proporcionar información relevante en este sentido, aunque debe señalarse que algunos suelos de la zona, debido a la acidez, no son muy aptos para la conservación de restos óseos 
"Junto a las puertas de sus casas, por grandeza, tienen dentro de la portada muchos pies de los indios que han muerto, y muchas manos; sin lo cual, de las tripas, porque no se les pierde nada, las hinchen de carne o de ceniza,..desto mucha cantidad; las cabezas, por consiguiente, tienen puestas, y muchos cuartos enteros. Un negro de un Juan de Cespedes...como viese estas tripas, creyendo ser longanizas, arremetió a descolgarlas para comerlas; lo cual hiciera si no estuvieran como estaban, tan secas del humo y del tiempo que habia que estaban alli colgadas. Fuera de las casas tienen puestas por orden muchas cabezas, piernas enteras, brazos, con otras partes de cuerpos, en tanta cantidad, que no se puede creer. Y si yo no hubiera visto lo que escribo, y supiera que en España hay tantos que lo saben y lo vieron muchas veces, cierto no contara que estos hombres hacían tan grandes carnicerias de otros hombres solo para comer; y asi, sabemos que estos gorrones son grandes carniceros de comer carne humana" (los subrayados son nuestros).

En esta cita, la ambigüedad es evidente. Uno bien puede leer o concluir que si ellos mataban y exhibían los cuerpos, ésto se debía a razones políticas y/o rituales, pero no por razones alimenticias.

Basándonos en la discusión anterior, bien parece que en lugar de un estado de guerra permanente motivado por la competencia por tierras de primera calidad - en respuesta a la presión demográfica- y de la práctica de un canibalismo con funciones alimenticias, tanto la guerra como el canibalismo en esta zona podrian ser entendidos mejor a partir de otra serie de razones o causas, como veremos a continuación.

Cameiro nos ofrece la posibilidad de explorar esta otra perspectiva. Argumenta él (1990:194) que la causa principal para ir a la guerra entre los cacicazgos del valle del Cauca parece haber sido la captura de prisioneros, los cuales fueron asesinados y/o sometidos a canibalismo en un contexto ritual (véase también Reichel-Dolmatoff 1984:80) o utilizados como esclavos en diferentes actividades. Dado que el análisis de las fuentes documentales no apoya la idea que el esclavismo fuera o tuviese un papel preponderante en el sistema económico de estos grupos (véase Escobar 1988) ${ }^{19}$, parecería enton-

19 Es importante anotar aqui que en cuanto al papel de la esclavitud entre estas comunidades, Carneiro (1991:175) lleva a los extremos la información presentada por Trimborn sobre el tema. Escobar (1988) ha discutido anteriormente el hecho de la evidencia sobre esclavitud y concluye, a mi parecer correctamente, que en lo que concierne al área del Valle Medio del río Cauca, la esclavitud no parece haber sido un elemento clave de la organización socioeconómica. Esto, por supuesto, no desconoce que la posesión de esclavos haya proporcionado una ventaja significativa a aquellos que los poseían sobre los que no. El punto a considerar es si la esclavitud como tal era la base 
ces que la explicación se encontrara en la esfera de lo ritual. Carneiro (1990:207) considera que aunque el canibalismo puede haber comenzado como una consecuencia de la guerra, éste se convirtió, a través del tiempo, en una causa para ella. El canibalismo llegó incluso a convertirse en un asunto gastronómico o de simple preferencia, según Carneiro (1990:2905). Mas categórico, Carneiro (1990:206) anota que el canibalismo fue una culminación lógica del complejo de guerra profundamente establecido que existía en el valle del Cauca.

No obstante, a la explicación anterior para la guerra sigue una serie de 'otras causas' tales como "el deseo de un cacique por expandir su dominio y adquirir el control sobre recursos naturales tales como minas de oro y depósitos de sal" (Carneiro 1990:193) o que,

"La conquista de territorios fue un incentivo particular de los cacicazgos de Guaca y Popayán, los cuales al momento del arribo de los españoles estaban activamente expandiendo sus territorios y, aparentemente, en camino a convertirse en estados" (Carneiro 1990:193,194).

Aunque tanto la captura de prisioneros como el control de recursos y la conquista de territorio, son causas para explicar la guerra, que contrastan con aquellas otras ya discutidas, debe indicarse aquí que Carneiro concibe el valle del Cauca en términos muy similares a los de Arocha. En realidad, Carneiro (1990:192; 1991:179) considera al valle del Cauca como una región de tierras agrícolas circunscritas. Esta circunstancia, por su parte, define lo que Carneiro considera fueron las condiciones bajo las cuales se originaron los cacicazgos y que dieron lugar al subsecuente desarrollo de los estados, esto es, crecimiento de población en el contexto de circunscripción ambiental y/o social que lleva directamente a una situación de presión demográfica y competencia por tierras agrícolas de primera clase.

Aunque Carneiro no expone (al menos no explícitamente) un argumento muy fuerte sobre presión demográfica en la zona como explicación causal del nivel de desarrollo alcanzado por los cacicazgos de Guaca y Popayán, se puede asumir que tal condición subyace o actúa como 'incentivo' en estos grupos para la conquista de territorios, puesto que esta es una condición básica de su

económica de estas sociedades. La evidencia sugiere que ese no fue el caso y que aún las finanzas de los caciques dependian fundamentalmente del trabajo o mano de obra de los miembros de su comunidad. 
modelo general. De lo contrario, se debería pensar que Carneiro estaría modificando su concepción original, esto es, considerando que el crecimiento de población no es la única variable capaz de producir o dirigir el proceso de complejidad social y por lo tanto, que existe más de un mecanismo que puede generar tal proceso. Si este fuese el caso, la guerra podria mantener el papel predominante que él le atribuye como mecanismo de evolución política, aunque la relación simple de causa-efecto entre población (densidad/tamaño) y complejidad social planteada anteriormente, no podría sostenerse más.

Es necesario aclarar aquí que Carneiro (1991:185) mismo ha señalado un cambio en su perspectiva. El considera ahora que,

“... seria de pronto necesario reconsiderar la noción -que yo mismo he sostenido anteriormente- de que el deseo de conquistar territorios es la causa más frecuente de la guerra entre cacicazgos. El hecho que las guerras de conquista emerjan al nivel de los cacicazgos y permanezcan como un rasgo común, no significa que TODAS las guerras al nivel del cacicazgo fueron motivadas por esta razón."

Por lo tanto, y aun tomando en consideración este cambio en perspectiva, me parece claro que la posición de Carneiro sobre la guerra en el valle del Cauca, particularmente su idea que ésta era de una naturaleza ritual, es al menos ambigua en relación con las otras 'causas' que él anota y, sobre todo, con relación a su modelo de evolución en general. Por una parte, lo que permanece como un cabo suelto en su argumento estriba en lo siguiente: después de todo, cómo es que la circunscripción actúa como una variable principal en la explicación de la naturaleza de los cacicazgos del valle del Cauca? (véase Carneiro $1990: 192 ; 1991: 170)$. Si la guerra no estuvo siempre motivada por la necesidad de adquirir tierras (como consecuencia del crecimiento demográfico), sino que pudo obedecer a un sinnúmero de causas, me parece que el papel que Carneiro le atribuye a la 'circunscripción' queda sin base. Más aun, si tal y como él ahora argumenta (Carneiro 1991:185), la evolución de los cacicazgos debe ser observada desde una perspectiva histórica de profundidad - ya que los cacicazgos pueden, y de hecho evolucionan, se consolidan y desintegranla interacción entre las múltiples ‘causas' de la guerra con 'circunscripción' y 'niveles de población', viene a convertirse, en lo que tiene que ver con el valle del Cauca, en una proposición sin mayores bases.

Por lo anterior, y en lo que tiene que ver estrictamente con el caso del valle medio del río Cauca, es claro que la información disponible no sustenta ningún argumento que trate de explicar la guerra, el canibalismo o aun el origen de los 
cacicazgos, como consecuencia de una interacción entre circunscripción y presión demográfica. Puesto que el valle del río Cauca no es una entidad homogénea (cultural, social o ecológica) se necesita de más estudios regionales que permitan evaluar el papel que estas variables jugaron en las diferentes subregiones (véase Schorr 1968). De prioridad absoluta, es la necesidad de un marco cronológico más preciso que el actual, de manera que los cambios en el medio ambiente, niveles de población, etc, puedan ser monitoreados.

Para concluir esta sección, podemos decir que si bien en principio los dos grupos de explicaciones discutidos (cf. Arocha vs. Carneiro) aparecen como claramente contrastantes, la verdad es, que observados detenidamente, comparten algunas de las ideas fundamentales en relación con el comportamiento de covarianza entre medio ambiente, población y guerra. Anotada esta similaridad, cabe entonces puntualizar que el problema fundamental que requiere una solución es precisamente el de medir estas variables en términos más precisos. Sólo de esta manera podrán hacerse evaluaciones consistentes de las explicaciones causales que se ofrecen. Sin lugar a dudas, los trabajos discutidos son puntos de partida importantes e indican claramente el potencial que tiene la región del valle del Cauca para profundizar en el estudio de la relación entre estas variables en el contexto más general de la evolución y desarrollo de las sociedades complejas.

\section{LA MATRIZ SOCIOPOLITICA DEL CANIBALISMO Y LA GUERRA: DOCUMENTOS ESPAÑOLES E INVESTIGACION ARQUEOLOGICA DE PATRONES DE ASENTAMIENTO}

La base de los estudios sobre la organización sociopolítica de las sociedades del valle del río Cauca, hasta el presente, es casi exclusivamente el análisis de documentos etnohistóricos ${ }^{20}$. Un problema que ésto conlleva es que los españoles no fueron consistentes en el uso de categorías o términos para describir las posiciones de rango o estatus, como tampoco las estructuras sociales en que se insertan las primeras. En este sentido, se podría argumentar que la falta de claridad obedece, al menos en parte, a la amplia gama de sociedades por ellos encontradas, así como a la variedad de formas como tales posiciones se expresaban.

\footnotetext{
20 La información más confiable que podemos tomar de las crónicas en cuanto a la guerra se refiere, es la restringida a las armas, rituales y sistemas defensivos. Estos han sido tantas veces descritos que no es necesario volver sobre ellos (véase Trimborn (1949) Carneiro (1990; Hernández (1948), Duque Gómez (1970)).
} 
Este problema puede ser ilustrado de la siguiente manera: Cieza (1985a:CXXX:44) anota que en el área de Popayán, "Todas estas vegas y valles fueron primero muy pobladas y sujetadas por el señor llamado Popayán, uno de los principales señores que hubo en aquellas provincias." No obstante, cuando Cieza compara los grupos de la provincia de Popayán - la cual en aquella época incluía una porción significativamente grande de la región del valle del Cauca - con aquellos del Perú, los primeros son descritos como 'behetrías' (Cieza 1985a:C-XIII:23), un término que se utilizó durante la época colonial para designar grupos que carecían de una jerarquía formal y de un cacicazgo hereditario ${ }^{21}$. La Lone (1982:296), al discutir el caso de los Incas ha expresado que,

“...nuestro conocimiento sobre la estratificación social ha sido obscurecido por la insistencia de los cronistas españoles en aplicar el término de cacique ó curacas indiscriminadamente a todos los lideres nativos desde aquellos de orden local hasta al propio Sapa Inca. Pero ahora, con el uso de documentos que fueron subestimados en el pasado tales como testamentos, reclamaciones y pleitos ...es posible mirar más en detalle a la configuración regional y local de estas jerarquías" 22 .

En nuestro caso, tal y como se discutió antes, aunque tal posibilidad puede estar aún vigente, nos parece que la manera más directa para enfrentar este problema son los estudios arqueológicos. Las posibilidades para un mejor entendimiento de este aspecto de las sociedades, están determinadas por nuestra habilidad para precisar las diferencias en escala entre estas sociedades, proporcionando asi unas condiciones más adecuadas para evaluar los argumentos que hasta el momento se han discutido.

Aunque no existe una definición de los cacicazgos sobre la cual exista consenso general (véase Earle 1989), parece que durante las últimas décadas ha surgido una serie de elementos alrededor de los cuales parece haber más acuerdo. Además del reconocimiento de la presencia de una gran variabilidad en el tamaño, así como en relación con otros elementos, entre las sociedades de tamaño mediano, como lo son los cacicazgos (véase Feinman y Neitzel 1984; Drennan 1987; Earle 1989), un elemento que parece ser clave es la existencia de un nivel de integración supracomunal (Carneiro 1981; Earle 1987).

\footnotetext{
21 Sobre la behetria, Tovar (1992:60) anota que ésta "no reproducia a una entidad sin estructura de poder, conforme lo definen las crónicas, sino a un tipo de organización con unas instituciones administrativas menos visibles e identificables para los europeos".

22 Ver Tovar $(1992: 60)$ en el mismo sentido.
} 
Se han hecho diferentes intentos para tratar el problema del grado de complejidad a partir de estudios arqueológicos de patrones de asentamiento, como mecanismo para establecer la existencia de jerarquías entre los asentamientos (cf. Pebles y Kus 1977; Paynter 1982). Es este aspecto precisamente el que queremos resaltar aquí como alternativa para solucionar, al menos en parte, los problemas que se presentan para el estudio de las sociedades del valle del Cauca y en el que los estudios arqueológicos podrían contribuir al proporcionar una línea de evidencia independiente para medir o establecer el tamaño y grado de complejidad de estas sociedades. Esta perspectiva de investigación se ilustrará mediante el análisis de los casos específicos de los Ansermas, Quimbayas y Armas.

Las crónicas indican que en la región de Anserma existían numerosos caciques menores pero sólo unos pocos principales (Sarmiento [1540] 1864:275). Entre estos caciques, es Ocuzca (Sardella [sf.] 1864:299; Sarmiento [1540] 1864:275-277) el que aparentemente desempeña un papel importante en términos de decisiones políticas de cobertura regional, tal y como se desprende, por ejemplo, de la descripción hecha por Sarmiento ([1540] 1864:276) sobre la captura de este cacique con el propósito de obligar a los otros caciques a aceptar la presencia española. Sarmiento ([1540] 1864:276) describe este personaje de la siguiente manera:

"Y este cacique es hombre algo en dias é gordo é de gran presencia, cuando habla, parece que asombra las gentes, tiene fama, por dicho de indios, de muy rico é lo debe ser segund su manera".

Sin embargo, esta descripción, particularmente en cuanto hace referencia a su 'riqueza', podría servir para tipificar a cualquier otro cacique de la región. En verdad, y de acuerdo con Robledo ([sf.] 1865:389) cualquier otro cacique en la zona parece haber sido tan poderoso o independiente como lo era Ocuzca.

Tomando en cuenta estas informaciones algo ambiguas, parecería que en el mejor de los casos, la situación en Anserma era la de una zona dividida en varias subregiones, cada una de ellas con su propio número de caciques menores y un cacique principal. Estos caciques principales, cada uno con residencia basada en un 'pueblo', ejercía control real sobre otros pueblos. Los pueblos fueron descritos como "juntos, las casas desviadas alguna distancia unas de otras " (Cieza 1985a:C-XV:26).

Entre los Quimbayas por su parte, la evidencia de la existencia de un nivel supracomunal de integración política, es más problemática. De acuerdo con 
diversas descripciones, existian entre 60 y 80 caciques, o aún más (compare Sarmiento [1540] 1864:286 con Robledo [sf.] 1865:398; véase también Friede 1982:24) pero sólo se reporta la presencia de cinco ó seis caciques principales (Robledo [sf.] 1865:399). Aunque Robledo ([sf.] 1865:398) establece que en Quimbaya los "señores eran más señores y los indios más obedientes" no hay indicación clara sobre el alcance del poder de estos caciques principales así como sobre las relaciones entre ellos mismos, con la excepción del reconocimiento que ellos eran amigos y confederados (véase Cieza 1985a:C-XXIV:35; Robledo [sf.] 1865:399).

El análisis hecho por Friede (1982:53-63) de los documentos concernientes a las llamadas Juntas de Guerra, reuniones de los Quimbayas para organizar una rebelión general, indican que la participación en éstas (aunque amplia y principalmente de carácter voluntario), fue también inducida mediante el envío de regalos y/o por intimidación. Este hecho sirve para indicar que, a pesar de las manipulaciones ejercidas por los caciques principales, muchos caciques menores disfrutaban cierto grado de autonomía. Así, mientras que en el caso de los Ansermas la información indica más claramente que en cuestiones de política regional la decisión de los caciques principales era el elemento clave, entre los Quimbayas el poderío de éstos parece haber sido menor (véase Duque 1970:64).

Entre los Quimbayas, la población también se concentraba alrededor de los pueblos de los caciques. La presencia de esta tendencia hace posible esperar que entre más densa la concentración de población, más grande fuese el poder de los caciques. Si los caciques principales citados entre los Quimbayas fueron en realidad poderosos, es de esperar que hubiera una densidad grande de población concentrada alrededor de sus domicilios (véase Drennan 1987).

El caso de los Armas nos presenta una situación que contrasta marcadamente con las dos anteriores en términos de organización social. En este caso, un solo individuo, Maitatama, es reconocido como el cacique principal de toda la región. Adicionalmente, el estatus de este personaje parece corr 2 lacionarse positivamente con el tamaño del asentamiento en que residía. Estó se desprende de la descripción proporcionada en el relato de Sarmiento ([1540] 1864:284) sobre la campaña española contra los Armas. Este cronista indica que antes de llegar a la aldea donde vivía Maitatama, los españoles tomaron posesión de varios pueblos en su viaje desde la provincia de Paucura. El primero de estos asentamientos, localizado en la zona limítrofe entre estos dos grupos, era pequeño; el segundo, ubicado más adentro en pleno territorio Arma fue también pequeño. Posteriormente llegaron a un tercero, localizado 
bien adentro en el territorio Arma y de tamaño más grande que los anteriores. Desde esta última localidad, pudieron observar en la distancia, el asentamiento que era el corazón del grupo Arma, el más grande de todos y el sitio donde vivía Maitatama (véase también Cieza 1985b:C-XI:171). De acuerdo con Oviedo (citado en Trimborn 1949:127) este asentamiento estaba conformado por cerca de 3.000 casas.

Esta clara correlación entre tamaño de los asentamientos y nivel de complejidad o jerarquía es también reforzado por otros elementos. Entre éstos, uno de los más interesantes e intrigantes es el de la composición de los individuos bajo el control de Maitatama. Aunque los españoles que viajaban en estas campañas no eran lingüistas, después de varios años muchos habían desarrollado cierta habilidad para percibir la presencia de variaciones lingüísticas. Cieza (1985a:C-XVIII:29) comenta que en la provincia de Arma habia tal variación lingüística, que en casi todo barrio o cima de colina se hablaba una lengua diferente. Bien sea que fuesen lenguas diferentes o sólo variaciones dialectales, lo importante para nuestra discusión es, que si hemos de aceptar las informaciones de los cronistas, debemos concluir a partir de esta información que en el caso de Maitatama, su poder no estaba reducido a un solo grupo humano unificado por la lengua.

Como último elemento vale la pena resaltar, en cuanto a la configuración del grupo Arma, que su ejército era de alrededor de 20.000 guerreros. Tomando entonces en consideración estas tres variables (jerarquía de asentamientos, composición multilingüística [dialectal y /o étnica] y tamaño del ejército) resulta claro que la posición de Maitatama es bien diferente de aquella que se infiere para los casos de los Ansermas y Quimbayas, respectivamente. El caso de los Armas, por su parte, nos proporciona un ejemplo de lo que de acuerdo con los modelos de evolución social, podría considerarse como un tipo de organización con las característica de un estado o, al menos, de 'protoestado' (véase Carneiro 1991:180).

No obstante, y como se anotó antes, el caso de los Armas es interesante pero también contradictorio. Nótese que Maitatama nunca fue descrito como un 'rey' ni tampoco existen indicaciones que a nivel de organización político/ administrativa, los Armas presentaran diferencias significativas al compararlos con los otros grupos de la región. Cieza (1985b:C-X:169) describe la provincia de Arma como "...la grande e muy riquísima provincia...que es la mayor y más poblada que hay en todo el Perú [sic]..". Mientras que la escasez de descripciones sobre organización social puede indicar que en realidad esta sociedad no era muy jerarquizada o al menos no muy diferente del grado al- 
canzado por otros grupos en el valle, debemos recordar que Cieza mismo, en unas instancias describe a Popayán como un caso de integración política compleja pero en otras, también como behetría. Mientras que esto sirve para establecer y reiterar la clase de problemas que las crónicas presentan, también nos sirve para preguntarnos entonces por qué, si en verdad Maitatama (y por extensión los Armas) fueron tan complejos, los españoles no describieron este grupo en más detalle?

Una posible explicación sería que los españoles quedaron cegados $\mathrm{y} / \mathrm{u}$ obsesionados con el espectáculo ofrecido por los guerreros Armas, quienes salieron al campo de batalla luciendo cascos y pectorales (armaduras) de oro (Sarmiento [1540] 1864:284; Cieza 1985a:C-XVIII:29; 1985b:C-XI:170), de ahí, pues, su nombre. Tales escenas pueden haber impactado tanto a los españoles que éstos perdieron todo interés en la descripción de aquellos aspectos que son nuestra preocupación ahora. No podemos desconocer aquí tampoco, que la resistencia de los Armas fue feroz (véase cita en página 18) ${ }^{23}$.

Los ejemplos anteriores ilustran cómo el estudio arqueológico de patrones de asentamiento puede contribuir al esclarecimiento de la organización política de estos grupos y así dilucidar las ambigüedades que los reportes españoles plantean. Mediante el estudio de los patrones de asentamiento, así como con la estimación de las poblaciones regionales y sus densidades (véase Drennan 1987, Drennan et al, 1991, Langebaek 1993) sería posible obtener líneas independientes para confrontar y evaluar las reconstrucciones hasta ahora avanzadas sobre los diferentes aspectos aquí discutidos de las sociedades del valle del Cauca.

No sobra enfatizar aquí, finalmente, que los estudios regionales deben ser complementados con estudios más detallados al nivel de los asentamientos específicos, así como de las unidades domésticas, para recobrar el tipo de información específica con la cual aportar elementos de juicio sobre aspectos como las dietas, intercambio de productos, diferencias económicas, etc ${ }^{24}$. En

23 Cabe anotar aqui que Tovar (1992:57-60) presenta algunos argumentos similares en torno a la configuración politica de los Armas, Quimbayas y la zona de Ancerma. No obstante, en nuestro caso, el tratamiento es diferente en tanto que lo que se explora es el aspecto de la distribución de la población con miras a sustentar el papel que los estudios de patrones de asentamiento pueden tener como herramientas analiticas.

24 Como ejemplo de esta perspectiva de análisis regional y sus subsequentes desarrollos, se puede ver los trabajos de Blick (1993), Taff(1993), Jaramillo (1994), Quattrin (1994) y Drennan y Quattrin (en prensa). 
este sentido, debe recalcarse que la información que tal tipo de estudios puede aportar para entender la guerra y aún el canibalismo, no se concibe como la recolección de pruebas directas, necesariamente, sino ante todo, como la recolección de información que permita hacer modelos con variables tales como la densidad de población por períodos específicos y sus variaciones, la capacidad de carga y los niveles de producción y así confrontar indirectamente las reconstrucciones y explicaciones vigentes en estas materias.

\section{CONSIDERACIONES FINALES}

Creemos que son claros los problemas que se derivan de una aceptación literal de las crónicas para reconstruir tanto los patrones de guerra, la organización social y aún el canibalismo entre las sociedades prehispánicas del valle del Cauca. De especial importancia es el problema que acarrea el desconocimiento del papel dinámico que la presencia española tuvo en la transformación de las estructuras sociales existentes, así como sobre las prácticas culturales. La presencia española generó cambios y creó nuevas situaciones políticas a las que los grupos indigenas debieron ajustarse.

El objetivo de este estudio no ha sido el de modificar la imagen sangrienta y caníbal que ha hecho famosas a las sociedades del valle del Cauca, sino, por el contrario, el de explorar los problemas que presentan las reconstrucciones y explicaciones vigentes para así, reflexionar sobre las formas más apropiadas de utilizar los documentos españoles, con el ánimo de entender la naturaleza de la guerra indígena, de la práctica del canibalismo y de la organización sociopolítica de estas comunidades. El desarrollo de estudios de carácter arqueológico regional sistemático como estrategia para evaluar la relación entre los patrones de asentamiento y el grado de organización política, ha sido discutido como una alternativa en tal sentido.

Si se considera a las sociedades del valle del Cauca en función de la presencia de conflicto tanto a nivel interno como externo, nos damos cuenta que estos grupos no son, después de todo, tan únicos o diferentes, especialmente una vez que se toman en consideración las imprecisiones e inconsistencias de las crónicas sobre tales aspectos, así como los problemas que se observan en las explicaciones basadas sobre estas. Si reconocemos que la guerra (y el conflicto en general) entre grupos vecinos fue seguramente una realidad (después de todo no habría razón para esperar que los grupos del valle del Cauca fuesen en este sentido diferentes de cualquier otro grupo humano pasado o presente [i, e, pacificos por definición, véase MaCauley 1990;13-16; Gregor 1990:106107]) debemos reconocer que los documentos españoles son a la vez una fuen- 
te importante de información y una fuente de problemas, la cual podemos utilizar con provecho si se diseñan estrategias de investigación apropiadas. Tal es el caso con la información sobre los patrones de asentamiento como se exploró en los casos de los Ansermas, Quimbayas y Armas. Esta creemos es una línea de investigación que vale la pena adelantar, para así tener una base apropiada para proponer y/o evaluar las reconstrucciones sobre los diferentes aspectos de estas sociedades y en esta medida, poder también contribuir a la evaluación de los modelos generales sobre desarrollo sociopolítico.

\section{BI BLIOGRA FIA}

ANONIMO

1886 Varias Noticias Curiosas sobre la Provincia de Popayán. En: Colección de Documentos Inéditos Relativos al Descubrimiento. Conquista y Colonización de las posesiones españolas en América y Oceania sacados en su mayor parte del real Archivo de Indias. Tomo V:487-493. Madrid: Imprenta de Manuel B. de Quiros.

ARENS, William

1979 The Man-Eating Myth: Anthropology and Anthropophagy. New York, Oxford University Press.

AROCHA, Jaime.

1987 Clima, Hábitat, Proteínas, Guerras y Sociedades del Siglo XVI. Arqueología, 3(1):28-42.

BALEE, William

1984 The ecology of Ancient Tupi Warfare. En: Warfare, Culture and Environment: 241-265 R. Brian Ferguson, ed. Orlando, Academic Press.

BLICK, Jeffrey P.

1987 The Cannibalism Issue: Structuralist and Materialist Interpretations and other concerns. Lambda Alpha Journal of Man, 18:122 .

1988

Genocidal Warfare in Tribal Societies as a Result of EuropeanInduced Culture Conflict. MAN (N.S.) 23(4):654-670. 
1993

Social Differentiation in the Regional Classic Period (A.D. 1900) in the Valle de la Plata. Colombia. Ph.D. Dissertation, Department of Anthropology, University of Pittsburgh.

BRAY, Warwick

1979 Gold of El Dorado. From the exhibition Gold of El Dorado: The Heritage of Colombia. American Museum of Natural History. New York, Henry N. Abrams, Inc, Publishers.

BROWN, Paula y Donald Tuzin, (eds.)

1983

The Ethnography of Cannibalism. Washington, D.C., Society for Psychological Anthropology.

BRUNHS, Karen Olson

1981 Prehispanic Ridged Fields of Central Colombia. Journal of Field Archaeology, 8:1-8.

CARNEIRO, Robert

1970

A Theory of the Origin of the State. Science 169:733-38.

1981

The Chiefdom: Precursor of the State. En: The Transition to Statehood in the New World.37-79, G. D. Jones and R. R. Kautz, eds. New York, Cambridge University Press.

1990

Chiefdom-level warfare as exemplified in Fiji and the Cauca Valley. En: The Anthropology of Warfare: 190-235. Jonathan Hass, ed. Cambridge, Cambridge University Press.

1991

The Nature of the Chiefdom as revealed by Evidence from the Cauca Valley of Colombia. En: Profiles in Cultural Evolution: Papers from a Conference in Honor of Elman R. Service: $167-$ 190, A. Terry Rambỏ and Kathleen Gillogly, eds. Anthropological Papers, Museum of Anthropology, University of Michigan. 
CASTILlO, Neila

1988 Complejos Arqueológicos y Grupos Etnicos del Siglo XVI en el Occidente de Antioquia. Boletin Museo del Oro 20:16-34. Bogotá.

CHAGNON, N. A.

1983 Yanomamo: the fierce people (3rd ed.). New York, Holt, Rinehart \& Winston.

1990

Reproductive and somatic conflicts of interest in the genesis of violence and warfare among tribesman. En: The Anthropology of War: 77-104. Jonathan Hass, ed. Cambridge University Press.

CIEZA DE LEON, Pedro

[1550]

$1985 \mathrm{a}$

La Crónica del Perú. En: Obras Completas, edición crítica, notas, comentarios e índices, estudios y documentos adicionales por Camelo Saenz de Santa María. Volumen 1. Madrid: Consejo Superior de Investigaciones Científicas Instituto "Gonzalo Fernández de Oviedo".

1985b Las Guerras Civiles Peruanas. En: Obras Completas,edición crítica, notas, comentarios e indices, estudios y documentos adicionales por Camelo Saenz de Santa María. Volumen 2. Madrid: Consejo Superior de Investigaciones Científicas Instituto "Gonzalo Fernández de Oviedo".

CUBILlOS, Julio César

1984 Asentamientos Prehispánicos en la Suela Plana del Río Cauca. Bogotá: Fundación de Investigaciones Arqueológicas Nacionales. Banco de la República.

DRENNAN, D. Robert

1987 Regional Demography in Chiefdoms. En: Chiefdoms in the Americas: 307-323, Robert D. Drennan and Carlos A. Uribe, eds. Lanham, MD. University Press of America. 
DRENNAN, D. Robert, Luis Gonzalo Jaramillo, Elizabeth Ramos, Carlos 1991 Augusto Sánchez, María Angela Ramírez y Carlos A. Uribe. Regional Dynamics of Chiefdoms in the Valle de la Plata, Colombia. Journal of Field Archaeology, 18:297-317.

DRENNAN, D. Robert and Dale W. Quattrin

En prensa Social Inequality and Agricultural Resources in the Valle de la Plata, Colombia. En Foundations of Social Inequality. Editado por T. Douglas Price and Gary M. Feinman. New York: Plenum.

DUQUE GOMEZ, Luis

1963 Los Quimbayas: Reseña Etnohistórica y Arqueológica. En: Historia de Pereira, Luis Duque Gómez; Juan Friede y Jaime Jaramillo, eds. Bogotá. Club Rotario.

1967 La Cuenca del río Cauca. En: Historia Extensa de Colombia, Volumen I, Tomo II. Academia de Historia Colombiana, Bogotá. Ediciones Lerner.

1970

Los Quimbayas. Bogotá: Imprenta Nacional.

EARLE, Timothy

1987 Chiefdoms in Archaeological and Ethnohistorical Perspective. Annual Review of Anthropology 16:279-308.

1989

The Evolution of Chiefdoms. Current Anthropology 30(1):8488 .

ECKERT, Georg

1945 El Culto a los Muertos y la Concepción de la Vida en el Valle del Cauca. Revista de Indias 19:73-122.

ESCOBAR DE, Geronimo

[1582] Relación sobre el carácter y costumbres de los Indios de la 1884 Provincia de Popayán. En: Colección de Documentos Inéditos Relativos al Descubrimiento, Conquista y Organización de las 
Posesiones Españolas en América y Oceania sacados en su mayor parte del Archivo de Indias. Tomo XLI, Madrid. Imprenta de Manuel B. de Quiros.

ESCOBAR, María Elvira

1988 Cacicazgos del Valle del Río Cauca: Señorío o Barbarie. En: Revista Colombiana de Antropologia 26:155-172.

FEINMAN, Gary y Jill Neitzel

1984 Too many types: an overview of sedentary pre-state societies in the Americas. En: Advances in Archaeological Method and Theory, Vol. 7: 39-102, edited by M. Shiffer. New York. Academic Press.

FERGUSON, R. Brian

1984 Introduction: Studying War. En: Warfare, Culture and Environment: 1-81, Ferguson R. Brian, ed. New York, Academic Press.

1990 Explaining War. En: The Anthropology of War: 26-55, Jonathan Hass, ed. Cambridge. Cambridge University Press.

FORD, James

1944

Excavations in the vicinity of Cali. Yale University Publications in Anthropology. No. 31. New Haven.

FRIED, Morton H., Marvin Harris and Robert Murphy et.al.,(eds.).

1968 War: The Anthropology of Armed Conflict and Aggression. New York: Natural History Press.

FRIEDE, Juan

1982

Los Quimbayas Bajo la Dominación Española: Estudio Documental. Bogotá; Carlos Valencia Editores.

1963 Los Quimbayas. Aportación documental al estudio de la demografia precolombina. Revista Colombiana de Antropología, Vol. XI pp.301-318. 
GREGOR, T.

1990 Uneasy peace: Intertribal relations in Brazil's upper Xingu. En: The Anthropology of War: 103-124, edited by J. Hass, Cambridge University Press, Cambridge.

HARNER, M. J.

1972 The Jivaro: people of the sacred waterfalls. Berkeley. University of California Press.

HARRIS, Marvin

1977 Cannibals and Kings, The origines of cultures. New York. Random House.

HARRISON, Thomas $\mathrm{H}$.

1948 Living Among Cannibals. London. George Harrap and Co. Ltd.

HERNANDEZ DE ALBA, Gregorio

1948 The Cultures of Northwest South America: Sub-Andean Tribes of the Cauca Valley. En: Handbook of South American Indians, Vol. 4: 297-327. Julian Steward, ed. Washington D. C., Smithsonian Institute.

HELMS, Mary W.

1976 Competition, Power and Succession to Office in Pre-Columbian Panama. En: Frontier Adaptations in Lower Central America:2535, Mary Helms and Francis L. Loveland (eds.). Philadelphia. ISHI.

1980

Succession to High Office in Pre-Columbian Circum-Caribbean Chiefdoms. Man 15(1):719-731.

HERRERA, Leonor, Marianne Cardale de Schrimpff y Warwick Bray $1990 \quad$ La arqueológia y el paisaje en la región Calima. En: Ingenierías Prehispánicas: 111-150; S. Mora (Ed.). Fondo FEN Colombia, ICAN, Bogotá.

JARAMILLO, Jaime

1964 La Población Indigena de Colombia en el momento de la conquista y sus transformaciones posteriores. Anuario Colombiano de Historia Social y de la Cultura 2:239-293. 
JARAMILLO, Luis Gonzalo

1991 Investigación Arqueológica en los municipios de Chinchiná, Villamaria, Palestina y Santa Rosa de Cabal. Boletín de Arqueología 4(1):29-40.

1994 The socioeconomic structure of Formative 3 communities in the Valle de la Plata. Colombia: Documenting the emergence of chiefdoms. Ph.D. Dissertation, Department of Anthropology, University of Pittsburgh.

JIJON Y CAAMAÑO

[1938] Sebastián de Belalcázar; Tomos I y II. Reimpreso como VII del 1983 Anuario Histórico Jurídico Ecuatoriano, Quito.

LA LONE, Darell

1982 The Incas as a Non-Market Economy: Supply on Command versus Supply and Demand. En: Context for Prehistoric Exchange:291-316, J. E. Ericson and T. K. Earle eds. New York. Academic Press.

LANGEBAEK, Carl H.

1992 Competencia por prestigio político y momificación en el Norte de Suramérica y el Istmo de Panamá. Revista Colombiana de Antropología, Volumen XXIX:9-26.

1993 From Hunters and Gatherers to Muiscas, Campesinos and Hacendados in the Eastern Higlands of Colombia: an archaeological survey in the Valles de Fuquene and Susa. Ph.D. Dissertation, Department of Anthropology, University of Pittsburgh.

\section{LLANOS, Héctor}

1981 Los Cacicazgos de Popayán a la llegada de los Españoles. Bogotá. Fundación de Investigaciones Arqueológicas Nacionales.

McCAULEY, Clark

1990

Conference Overview. En: The Anthropology of War: 3-25, editado por J. Hass. Cambridge University Press, Cambridge. 
PADILLA, Silvia

1977 Tasaciones de Encomiendas de Popayán en el siglo XVI. En: La Encomienda en Popayán: Tres Estudios, S. Padilla, L. López y L. González, eds. Sevilla: Escuela de Estudios Hispano-Americanos, Consejo Superior de Investigaciones Científicas.

PAYNTER, R.

1982 Stratification and Spatial Organization. En: Models of Social Inequality: Settlement Patterns in Historical Archaeology:21-45, editado por Robert Paynter. New York. Academic Press.

PEEBLES, Christopher S. y Susan M. Kus

1977 Some Archaeological correlates of Ranked Societies. American Antiquity, 42(3):421-48.

PINEDA CAMACHO, Roberto

1987 Malocas de Terror y Jaguares Españoles: Aspectos de la resistencia Indígena del Cauca ante la invasión Española del siglo XVI. Revista de Antropología, 3(2): 87-114.

QUATTRIN, Dale

1994 Interaction and Exchange during the Formative Period (1000 B.C. - A.D. I), in the Valle de la Plata, Colombia. Ph.D. Dissertation, Department of Anthropology. University of Pittsburgh.

REICHEL-DOLMATOFF, Gerardo

1984 Colombia Indígena, Período Prehispánico. En: Manual de Historia de Colombia. Vol. I: 33-115. Bogotá. Colcultura.

RESTREPO TIRADO, Ernesto

1912 Ensayo Etnográfico y Arqueológico de la Provincia de los Quimbayas en el Nuevo Reino de Granada. Boletín de Historia y Antigüedades 7(80):465-490; 7(80): 539-546; 7(80):593615. Bogotá: Academia Nacional de Historia.

ROBLEDO, Jorge

[sf.] Descripción de las Provincias de Anserma. En: Colección de 1865 Documentos Inéditos Relativos al Descubrimiento, Conquistay Organización de las posesiones Españolas en América y Oceanía 
sacados en su mayor parte del Real Archivo de Indias, Tomo III: 389-413, Madrid: Imprenta de Manuel B. de Quiros.

SALGADO, Héctor

1986 Asentamientos Prehispánicos en el Noroccidente del Valle del Cauca. Bogotá: Fundación de Investigaciones Arqueológicas Nacionales.

SARDELLA, Juan Baptista

[sf.] Relación del Descubrimiento de la Provincia de Antiochia por 1864 Jorge Robledo. En: Colección de Documentos Inéditos Relativos al Descubrimiento. Conquista y Organización de las Posesiones Españolas en América y Oceanía sacados en su mayor parte del Archivo de Indias, Tomo II: 291-356, Madrid. Imprenta de Manuel B. de Quiros.

SARMIENTO, Pedro

[1540] Relación del Viaje del Capitán Jorge Robledo a las Provin1864 cias de Ancerma y Quimbaya. En: Colección de Documentos Inéditos Relativos al Descubrimiento, Conquista y Organización de las Posesiones Españolas en América y Oceanía sacados en su mayor parte del Archivo de Indias, Tomo II: 267-291. Madrid. Imprenta de Manuel B. de Quiros.

SCHORR, Thomas S.

1965 Cultural Ecological Aspects of Settlement Patterns and Land Use in the Cauca Valley, Colombia. Doctoral Dissertation, Tulene University. Ann Arbor: University Microfilms International (1984).

1968

Cauca Valley Settlements, A Culture Ecological Interpretation. En: XXXVII Congreso Internacional de Americanistas, Memorias 1:449-466. Buenos Aires (1966).

1970

Fighting and Killing Behavior Between Human Populations: A Reflection of the Current Controversies. Ponencia presentada al XXXIX Congreso Internacional de Americanistas. Lima: Agosto 5 . 
TAFT, Mary

1993 Patterns of Ceramic Production and Distribution (Patrones de Producción y Distribución de la Cerámica). En: Prehispanic Chiefdoms in the Valle de la Plata, Volume 2: CeramicsChronology and Craft Production (Cacicazgos Prehispánicos del Valle de la Plata. Tomo 2: Cerámica-Cronología y Producción Artesanal), editado por Robert D. Drennan, Mary M. Taft y Carlos A. Uribe. University of Pittsburgh y Departamento de los Andes, Pittsburgh y Santafé de Bogotá; pp:103-185.

TANNAHILL, Reay

1975 Flesh and Blood: A history of the Cannibal Complex, New York. Stein and Day.

TOVAR, Hermes

1970 Estado Actual de los Estudios de Demografía Histórica en Colombia. Anuario Colombiano de Historia Social de la Cultura, 5:65-111.

1992

Colombia: lo diverso, lo múltiple y la magnitud dispersa. Maguaré, 8:47-79. (Este texto aparece también en Relaciones y $\mathrm{Vi}$ sitas a los Andes $S X V I$. Colección de Historia de la Biblioteca Nacional, Colcultura y el Instituto de Cultura Hispánica 1993).

TURNEY-HIGH, Harry H.[1949]

1971 Primitive War: Its Practice and Concepts. Columbia. University of South Carolina Press.

TRIMBORN, Hermann

1949

Señorío y Barbarie en el Valle del Cauca. Madrid. Consejo Superior de Investigaciones Cientificas Gonzalo Fernández de Oviedo.

VALENCIA, Alfonso

1991 Resistencia Indigena a la Colonización Española. Cali. Universidad del Valle.

ZUDEIMA, R. Tom

1983 Hierarchy and Space in Incaic Social Organization. Ethnohistory $30(2): 49-75$. 